\title{
Bitterstoffe - von der traditionellen Verwendung bis zum Einsatz an der Haut
}

Ute Wölfle, Christoph M. Schempp

Klinik für Dermatologie und Venerologie/

Forschungszentrum skinitial, Universitätsklinikum Freiburg

\section{ZUSAMMENFASSUNG}

Bitterstoffe werden in der traditionellen Phytotherapie schon lange zur Linderung von Magenbeschwerden sowie bei Gallen- und Verdauungsproblemen eingesetzt. Erst seit wenigen Jahren weiß man allerdings, dass Bitterstoff-Rezeptoren außer in den Geschmacksknospen der Zunge auch im gesamten Verdauungstrakt und in nahezu allen anderen extraintestinalen Organen gebildet werden. In diesem Beitrag werden geschichtliche, pharmakologische, medizinische und neueste naturwissenschaftliche Erkenntnisse zu den Bitter- stoffen und Bitterstoff-Rezeptoren mit besonderem Augenmerk auf die Bedeutung der Bitterstoff-Rezeptoren für die Haut aufgezeigt. Bitterstoffe aus dem Gelben Enzian, z. B. Amarogentin, binden an Bitterstoff-Rezeptoren der Haut, es kommt in den Keratinozyten zu einem Einstrom von Kalzium und zur Bildung von Proteinen und Lipiden, die an der Ausbildung der Hautbarriere beteiligt sind. Somit beleben Bitterstoffe den Stoffwechsel der Haut und regenerieren die Hautbarriere. Dies kann für die Behandlung von Hautkrankheiten mit einer Barrierestörung, wie z. B. bei der Neurodermitis oder sehr trockener Haut, genutzt werden.
Bitterstoffe dienen Pflanzen u.a. als Abwehrsubstanz gegen Fressfeinde. Leiden pflanzenfressende Tiere, wie z. B. Schafe, allerdings an Übelkeit, fressen sie die bitter schmeckende Schafgarbe, obwohl sie sie sonst auf der Weide stehen lassen. Schimpansen in Uganda, die an Malaria erkrankt sind, heilen sich auch mit Bitterstoffen. Sie fressen dann die extrem bitteren Blätter des Mahagoni-Gewächses Trichiliar rubescens. Die Inhaltsstoffe wirken dabei ähnlich wie Chloroquin, ein gängiges Malariamittel des Menschen [1]. Die Schimpansen nutzen allerdings nicht nur die Blätter einer Pflanze, sondern fressen verschiedene Arten. Eine weitere Pflanze ist z.B. der Mjonso-Baum (Vernonia amygdalina), den Schimpansen normalerweise wegen seiner bitteren und giftigen Blätter nicht fressen [2], [3]. Doch an Malaria erkrankte Tiere fressen die Blätter oder lutschen das Mark aus den Zweigen. In diesen Pflanzenteilen konnten Steroidglykoside mit antibakteriellen und antiparasitischen Eigenschaften gefunden werden [4]. Dadurch, dass mehrere verschiedene Pflanzen gefressen werden, ist es für den Malariaerreger schwieriger, Resistenzen zu entwickeln - ein häufiges Problem, das beim Menschen u.a. bei der Malariabekämpfung auftritt [5].

Der Bitterblattbaum Mjonso wird auch von den Stammesmitgliedern der WaTongwe in Zentralafrika eingesetzt. Dieses Naturvolk kocht die Blätter und isst sie mit Fleisch, um Magenbeschwerden, Malaria und Darmparasiten zu bekämpfen [5].

\section{Traditionelle und neuzeitliche Nutzung von Bitterstoffen}

In der ca. 5000 Jahre alten ayurvedischen Medizin spielen bitter schmeckende Heilkräuter eine wichtige Rolle, insbesondere in der richtigen Ernährung. „Bitterstoffe reduzieren nach ayurvedischer Auffassung Körpergewebe, v. a. Fett (Meda), reinigen das Blut (Rekta) und tonisieren Muskelgewebe (Mamsa)“ [6]. Ebenso haben Bitterstoffe als fester Bestandteil ausgewogener Ernährungskonzepte eine lange Tradition in der chinesischen Medizin: „Der bittere Geschmack ist dem Element Feuer und somit der Hitze, dem Intellekt, dem Handeln, der Freude, dem Lachen und dem Wachstum zugeordnet“ [7].

Vom griechischen Arzt Hippokrates (460-360 v. Chr.) über Hildegard von Bingen (1098-1179) bis ins Spätmittelalter zu Leonhart Fuchs (1502-1566) nahmen bitter schmeckende Pflanzen in den Kräuterbüchern einen wichtigen Platz ein [8]. Unter den zahlreichen Hinweisen gegen innere Leiden wurde besonders die fiebersenkende Wirkung hervorgehoben. Dies hat sich bei einigen Drogen noch in der Namensgebung erhalten: Centaurium umbellatum heißt auch Fieberkraut, und Menyanthes trifoliata Fieberklee.

Mit der Entwicklung erster moderner Analysenmethoden in den Naturwissenschaften begann die Identifizierung und Isolierung bitter schmeckender Komponenten in Heilkräutern. Deshalb gab es Anfang des 20. Jahrhunderts ein 
umfangreiches beschreibendes Wissen über Bittersubstanzen. Über die physiologische Wirkung der Bitterstoffe war jedoch so gut wie nichts bekannt. Die medizinische Bedeutung der Bitterstoffe ging in den nächsten Jahrzehnten immer weiter zurück und Bitterstoffe hatten nur noch eine gewisse Bedeutung zur Appetitanregung in Form von bitteren Aperitifs oder zur Verdauungsförderung als Magenbitter [9].

Gleichzeitig wurden aus den meisten Gemüsesorten und Früchten Bitterstoffe zugunsten eines angenehmeren und milderen Geschmacks herausgezüchtet. In den letzten Jahren erlebten Bitterstoffe allerdings eine Renaissance aufgrund der vielfach beschriebenen positiven Eigenschaften, die in den folgenden Abschnitten näher beschrieben werden.

\section{Biochemische Struktur von Bitterstoffen}

Bitterstoff-Heilpflanzen werden heute aufgrund ihrer Inhaltsstoffe oder ihrer sensorischen Qualitäten in verschiedene sogenannte Amara eingeteilt ( $\triangleright$ Tab. 1) [10]. Chemisch gehören pflanzliche Bittersubstanzen zu unterschiedlichen Stoffgruppen: Es finden sich Monoterpene, Sesquiterpene, Diterpene, Triterpene, Iridoidglykoside, Secoiridoidglykoside, Phloroglucinderivate, Peptide, Aminosäuren und Alkaloide [10]. Als Bitterstoffe werden dabei alle Substanzen zusammengefasst, die bitter schmecken, unabhängig von ihrer chemischen Struktur [11]. Eindrücklich sieht man dies am Beispiel des Tryptophans, das in der L-Form bitter schmeckt und ein Bitterstoff ist, während das Enantiomer in der D-Form süß schmeckt und deshalb zu den Süßstoffen zählt [12]. Es gibt somit keine einheitliche chemische Struktur, an der man den bitteren Geschmack ablesen oder gar vorhersagen könnte.

Die Bitterqualität kann deshalb nur durch Ermittlung des Bitterwertes bestimmt werden. Dazu wird die subjektive bittere Empfindlichkeit zu der Referenzsubstanz Chininhydrochlorid bei Prüfpersonen ermittelt. Der Bitterwert einer wässrigen Lösung von Chininhydrochlorid beträgt 200000 . Das bedeutet, dass für Prüfpersonen 1 Gramm Chininhydrochlorid in 200 Liter Wasser gelöst gerade noch bitter schmeckt [13]. Der Bitterwert entspricht dann dem Kehrwert der Verdünnung eines Stoffes, einer Flüssigkeit oder eines Extraktes, die für die Prüfperson gerade noch bitter schmeckt. Auf diese Weise lassen sich Bitterstoffdrogen nach ihren Bitterwerten einordnen ( Tab.2). Nach diesem Verfahren gilt der Gelbe Enzian, Gentiana lutea, als eine der bittersten bekannten Pflanzen. Sie ist in den Mittelgebirgen und den Alpen beheimatet. Es handelt sich um eine mehrjährige, bis zu $1,5 \mathrm{~m}$ hohe Pflanze mit großen, goldgelben in Trugdolden
>Tab. 1 Einteilung der bitteren Pflanzen (Amara) (modifiziert nach [10]).

\begin{tabular}{|c|c|}
\hline Bezeichnung und Kriterium & Beispiele \\
\hline Amara pura (tonica) & $\begin{array}{l}\text { Enzianwurzel, Bitterholz, Bitter- } \\
\text { klee, Tausendgüldenkraut, Arti- } \\
\text { schocke, Löwenzahnwurzel }\end{array}$ \\
\hline $\begin{array}{l}\text { Amara aromatica (Bitterstoffe und } \\
\text { ätherisches Öl) }\end{array}$ & $\begin{array}{l}\text { Angelikawurzel, Hopfenzapfen, } \\
\text { Orangenschalen, Wermutkraut, } \\
\text { Kardobenediktenkraut, } \\
\text { Schafgarbenkraut }\end{array}$ \\
\hline $\begin{array}{l}\text { Amara adstringentia (Bitterstoffe und } \\
\text { Gerbstoffe, Tannine) }\end{array}$ & $\begin{array}{l}\text { Chinarinde, Condurangorinde, } \\
\text { Bitterklee }\end{array}$ \\
\hline $\begin{array}{l}\text { Amara mucilaginosa (Bitterstoffe und } \\
\text { bedeutsame Mengen Schleimstoffe) }\end{array}$ & Isländisches Moos \\
\hline $\begin{array}{l}\text { Amara acria (Bitterstoffe und bedeut- } \\
\text { same Mengen Scharfstoffe) }\end{array}$ & $\begin{array}{l}\text { Ingwerwurzel, Kalmuswurzelstock, } \\
\text { Gelbwurz }\end{array}$ \\
\hline
\end{tabular}

stehenden Blüten, die in mehreren Stockwerken aus den Blattachseln wachsen (•Abb. 1). Das in den Wurzeln von Gentiana lutea in einer Konzentration von 0,02-0,04\% enthaltene Amarogentin gilt als die bitterste natürliche Substanz mit einem Bitterwert von 58000000 (> Tab. 2) [10].

- Tab. 2 Beispiele für Bitterwerte (nach [10]).

\begin{tabular}{|l|l|}
\hline Substanz/ Droge & Bitterwert \\
\hline Amarogentin & 58000000 \\
\hline Enzianwurzel & $10000-25000$ \\
\hline Wermutkraut & $10000-25000$ \\
\hline Fieberkleeblätter & $4000-10000$ \\
\hline Tausendgüldenkraut & $2000-10000$ \\
\hline Pomeranzenschalen & $1000-2500$ \\
\hline Benediktenkraut & $1000-2500$ \\
\hline Chinarinde & 1000 \\
\hline Löwenzahnwurzel & $>100$ \\
\hline
\end{tabular}

\section{Pharmakologische Wirkungen von Bitterstoffen}

Bitterstoff-Pflanzen bzw. ihre Extrakte gelten als appetitanregend, sekretionsfördernd und darmmotilitätssteigernd. Sie werden verwendet zur Behandlung von Appetitlosigkeit, dyspeptischen Beschwerden bzw. Magen-Darm-Beschwerden und teilweise auch bei Störungen des Galleflusses [10]. In der Phytotherapie werden den Bittermitteln zusätzlich antidepressive und konstitutionsfördernde Wirkungen zugeschrieben. Mit Erfolg werden sie auch bei Müdigkeit, Erschöpfung, Stress oder allgemein somatoformen Störungen eingesetzt [10]. 


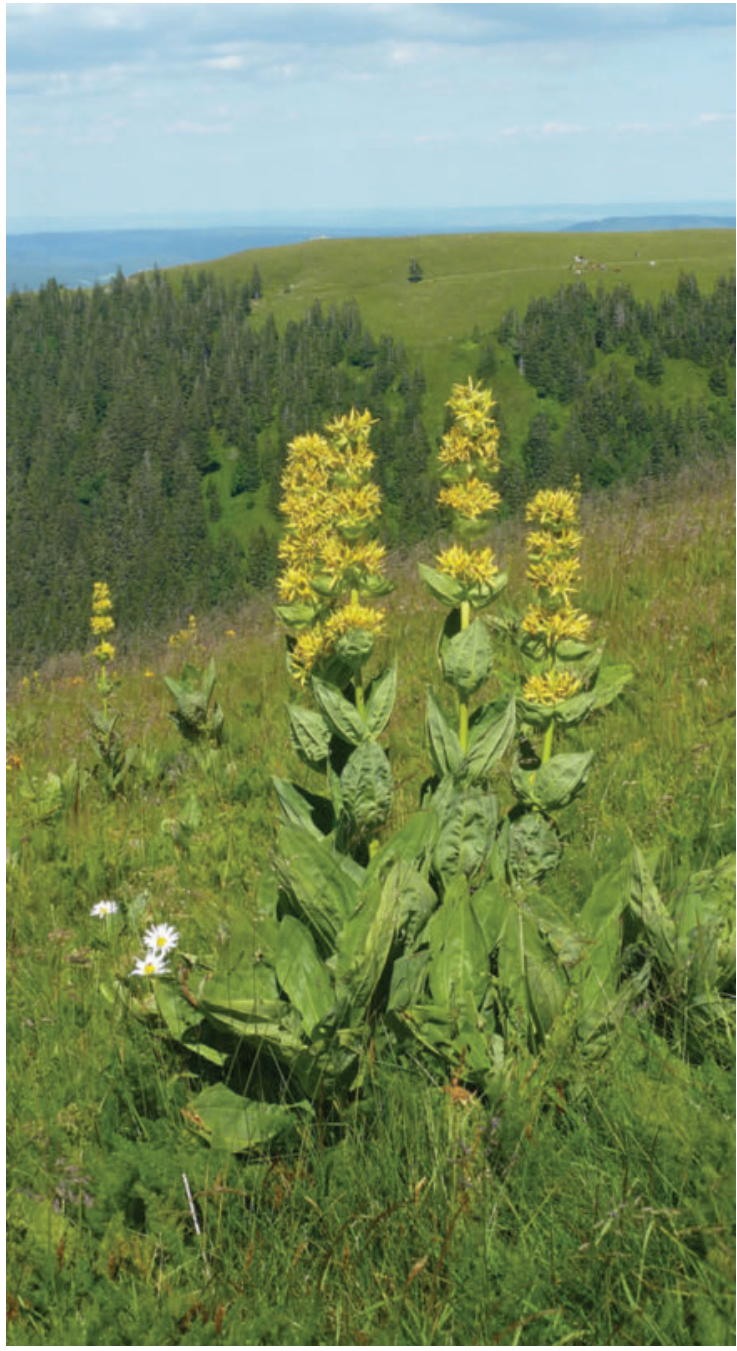

Abb. 1 Gelber Enzian (Gentiana lutea L.) am Feldberg. Foto: Stefan Martin

Für einzelne bitter schmeckende Bitterstoff-Heilpflanzen sind weitere therapeutische Möglichkeiten aufgezeigt worden. Beispielhaft werden hier die Bitterstoffdrogen Wermut (Artemisia absinthium) und Artischocke (Cynara scolymus) genannt. Wermut zeigte in einer Studie von 2007 eine antientzündliche, steroidmindernde, symptomlindernde (u.a. auch antidepressive) Wirksamkeit bei Patienten mit Morbus Crohn [14]. Artischockenblätterextrakte wiesen in verschiedenen Studien antibakterielle, antivirale (HIV), hepatoprotektive bzw. -stimulierende, cholesterinsenkende, choleretische, diuretische und antioxidative Effekte auf [15], [16]. Der Anteil der Bitterstoffe an diesen Wirkungen lässt sich aber nicht genau beurteilen, weil die Artischocke auch relativ hohe Konzentrationen von pharmakologisch wirksamen Flavonoiden enthält.

Innerlich angewendet, spielen Bitterstoffe vor allem in der anthroposophischen Medizin (AM) eine Rolle, z. B. bei der Therapie von Hauterkrankungen und Atopien. So spielt die Behandlung der Haut über die Anregung der
Verdauung, speziell der Lebertätigkeit, eine wichtige Rolle in der AM. Eine Reihe von sog. „Leberdermatosen“ (v. a. Akne und Rosazea) werden mit bitteren Pflanzenstoffen, z. B. aus dem Löwenzahn (Taraxacum officinale) behandelt [17].

\section{Entdeckung der Bitterstoff-Rezeptoren und ihre physiologische Bedeutung}

Obwohl Bitterstoffe schon seit vorchristlicher Zeit als appetitanregende und tonisierende Heilmittel eingesetzt wurden, war bis Ende des letzten Jahrhunderts kaum etwas über die Physiologie des Schmeckens bekannt. Man wusste zwar, dass sich das Schmecken auf der Zunge und im Mund konzentriert. Aber erst 1992 gelang es, ein Signaltransduktionsprotein nachzuweisen, das in Geschmackszellen spezifisch exprimiert wird - das Gustducin, ein trimeres G-Protein ( $G \alpha \beta \gamma$ ) [18], [19]. Erst im Jahr 2000 entdeckte der Molekularbiologe Jayaram Chandrashekar die Gene für die Bitterstoff-Rezeptoren $(T 2 R)$ [20]. In den folgenden Jahren wurden 25 dieser Rezeptoren beim Menschen beschrieben. Diese Rezeptoren sind hochkonserviert und werden sowohl in Insekten (Drosophila) als auch in Vertebraten exprimiert [21].

Nach Bindung von Bitterstoffen an den entsprechenden Rezeptor kommt es nach Signalweiterleitung über Gustducin zur Aktivierung der Phospholipase C- $\beta 2$ (PLC- $\beta 2$ ) und schließlich zur Öffnung des Kationenkanals TRPM5. Der Einstrom von Natriumionen in die Zelle bewirkt eine Depolarisation der Geschmackszelle und Signalweiterleitung ins Gehirn, wo das Geschmacksempfinden ausgelöst wird [22]. Bitterstoff-Rezeptoren werden außerhalb des oberen Gastrointestinaltraktes in der glatten Muskulatur der Bronchien [23], im respiratorischen Flimmerepithel [24], in den epithelialen Zellen des Magens, des Darms und der Blase [25], im verhornenden Epithel der Haut [26], in den Spermien [27] und im Gehirn [28] gebildet.

Abhängig vom jeweiligen Zelltyp kommt es nach Rezeptoraktivierung zur Vasodilatation (glatte Muskelzellen der Bronchien), zur NO-Sekretion und Erhöhung der Schlagfrequenz der Zilien (respiratorisches Flimmerepithel), zur Ausschüttung von gastrointestinalen Peptiden (Magen-Darm-Trakt) oder zur Steigerung des Neuritenwachstums (Gehirn) [29].

\section{Bitterstoffe für die Haut}

Bei vielen Hautkrankheiten kommt es zu Entzündungen und zu einer Schädigung der Epidermis, sodass die Haut keinen vollständigen Barriereschutz mehr besitzt. Dies begünstigt wiederum ein Austrocknen der Haut und dadurch Juckreiz und Anfälligkeit für bakterielle Infektionen. Pflanzliche Bitterstoffe, z.B. Amarogentin aus dem 
Gelben Enzian und Salicin aus der Weidenrinde, binden an Bitterstoff-Rezeptoren der Haut und induzieren einen Kalzium-Einstrom. In der Folge kommt es zur Bildung von Proteinen, die beim Aufbau der Hautbarriere eine wichtige Rolle spielen, z. B. Keratin 10, Involukrin und Filaggrin [26]. Bitterstoffe des Gelben Enzians regen auch die Synthese von Hautlipiden in Keratinozyten an ( den Hautlipiden gehören freie Fettsäuren, Cholesterin und Ceramide [30]. Dabei ist Ceramid 3 besonders wichtig für die Ausbildung der Hautbarriere. Dies zeigt sich daran, dass bei entzündlichen Hautkrankheiten mit beeinträchtigter Hautbarriere, wie der Neurodermitis und Psoriasis, Ceramid 1 und 3 vermindert vorliegen [31], [32].

Dabei ist besonders bemerkenswert, dass ein Extrakt aus dem Gelben Enzian die Lipidsynthese im Stratum corneum in einem prospektiven placebokontrollierten Halbseitenvergleich am Unterarm bei 33 Probanden statistisch relevant erhöhen konnte [30]. Der Enzian-Extrakt könnte auch bei Patienten mit atopischer Dermatitis die Lipidsynthese anregen und dadurch möglicherweise die Krankheitssymptome verbessern. Dabei würden besonders die Hautregionen von einer topischen Behandlung mit dem Enzian-Extrakt profitieren, in denen die Lipide ausschließlich von epidermalen Zellen gebildet werden, z. B. im Bereich der Lippen, in den Armbeugen und den Kniekehlen. Interessanterweise sind gerade diese Körperregionen besonders stark von atopischer Dermatitis betroffen. Dieser Effekt könnte auch bei trockener Altershaut genutzt werden, denn während der Hautalterung vermindert sich die Ceramidkonzentration im Stratum corneum ab dem Alter von 20 Jahren um etwa 10-15\% alle 10 Jahre [33].

Bitterstoffe wirken in der Haut aber nicht nur direkt auf die Keratinozyten, sondern haben auch eine immunmodulierende Wirkung im Zusammenspiel mit Mastzellen und Keratinozyten. Amarogentin hemmt die SubstanzPinduzierte Produktion des Entzündungsmediators TNF- $\alpha$ in humanen Mastzellen. Außerdem reduziert es in Keratinozyten die TNF- $\alpha$ - und Histamin-induzierte IL-8 und MMP-1-Expression, wodurch die Einwanderung von Entzündungszellen abnimmt. Diese Wirkung ist vergleichbar mit Azelastin, einem gängigen Antihistaminikum [34].

Mastzellen, T-Zellen und Keratinozyten befinden sich während einer Infektion in enger Nachbarschaft in der obersten Schicht der Dermis und beeinflussen so die Entzündung. Bitterstoffe wie Amarogentin modulieren möglicherweise das Milieu der entzündeten Haut und wirken auf diese Weise antientzündlich. Deshalb könnte eine Therapie mit Bitterstoffen wie Amarogentin auch bei TNF- $\alpha$ - und Histamin-induzierten entzündlichen Hauterkrankungen wie der chronischen atopischen Dermatitis und der Urtikaria (Nesselsucht) unterstützend wirken.

Darüber hinaus ändert sich die Expression von BitterstoffRezeptoren in Abhängigkeit vom Lebensalter. Während

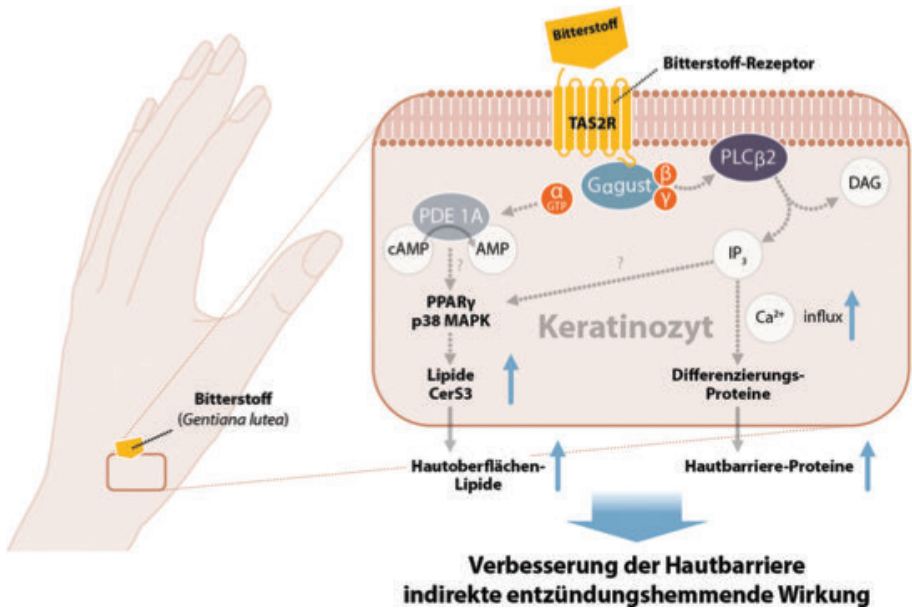

- Abb. 2 Zusammenfassendes Schema der innovativen Wirkung von Bitterstoffen für die Haut am Beispiel von Enzianextrakt. Graphik: Matthias Emde

junge Keratinozyten nur eine schwache Färbung des Bitterstoff-Rezeptors T2R1 zeigen, ist diese Färbung in alten Keratinozyten deutlich verstärkt, das heißt, alte Keratinozyten besitzen mehr Bitterstoff-Rezeptoren. Dies weist darauf hin, dass gerade auch ältere Personen von einer topischen Bitterstofftherapie profitieren könnten [35]. Es könnte sein, dass die Haut im Alter verstärkt BitterstoffRezeptoren bildet, um der dünner werdenden Hornschicht der Haut entgegenzuwirken. Denn durch die verstärkte Bildung der Bitterstoff-Rezeptoren können die körpereigenen Liganden (vor allem Aminosäuren) und Bitterstoffe aus der Nahrung besser ausgenutzt werden. Auch topisch applizierte Bitterstoffe binden an die Rezeptoren der Epidermis. Somit können Bitterstoffe bei der Pflege von Altershaut und von trockener Haut von Nutzen sein.

\section{FAZIT}

Insgesamt sind Bitterstoffe wie Amarogentin durch ihre hautregenerierende und barrierestärkende Wirkung für die Behandlung von Hautproblemen mit Barrierestörungen und reduziertem Lipidgehalt geeignet. Diese liegen besonders bei der Neurodermitis und sehr trockener Haut, z. B. Altershaut, vor. Doch auch bei Histamin-induzierten Hautkrankheiten, wie sie bei der chronischen Dermatitis und der Urtikaria vorliegen, könnten Bitterstoffe eine zusätzliche Therapieoption darstellen.

\section{ABSTRACT}

Bitter substances: From medical history to a rational dermal treatment option

Bitter substances have been used for a long time to treat gastric disorders as well as bile and digestion problems. Only recently it was discovered that bitter taste 
receptors were expressed not only in the taste buds of the tongue but throughout the digestive tract and in almost all extraintestinal organs. In this review historical, pharmacological, medical and the most recent scientific discoveries to bitter compounds and bitter taste receptors were considered with focus on bitter taste receptors in the skin. Bitter compounds of Gentiana lutea, e.g. amarogentin, bind to bitter taste receptors in the skin. This leads to calcium influx into the cell and the production of proteins and lipids that are involved in the formation of the skin barrier. In this way bitter compounds promote the regeneration of the skin barrier. Bitter substances might be used to improve skin disorders with an impaired epidermal barrier, e. g. very dry skin and atopic eczema.

\section{Interessenkonflikt}

Die Autoren haben Patente auf die topische Anwendung von Bitterstoffen und erhalten Lizenzen für die Produkte der Firma Velan Skincare.

\section{Korrespondenzadresse}

Priv.-Doz. Dr. rer. nat. Ute Wölfle

Universitätsklinikum Freiburg

Klinik für Dermatologie und Venerologie

Profilgruppe Entzündliche Hauterkrankungen

Forschungszentrum skinitial

Hauptstraße 7

79104 Freiburg

ute.woelfle@uniklinik-freiburg.de

\section{Literatur}

[1] Krief S, Martin MT, Grellier P et al. Novel antimalarial compounds isolated in a survey of self-medicative behavior of wild chimpanzees in Uganda. Antimicrob Agents Chemother 2004; 48: 3196-3199. doi:10.1128/AAC.48.8.3196-3199.2004

[2] Koshimizu K, Ohigashi H, Huffman MA. Use of Vernonia amygdalina by wild chimpanzee: possible roles of its bitter and related constituents. Physiol Behav 1994; 56: 1209-1216

[3] Huffman MA. Animal self-medication and ethno-medicine: exploration and exploitation of the medicinal properties of plants. Proc Nutr Soc 2003; 62: 371-381

[4] Jisaka M, Kawanaka M, Sugiyama $\mathrm{H}$ et al. Antischistosomal activities of sesquiterpene lactones and steroid glucosides from Vernonia amygdalina, possibly used by wild chimpanzees against parasite-related diseases. Biosci Biotechnol Biochem 1992; 56: 845-846

[5] Vaas R. Affen weisen den Weg zu neuen Wirkstoffen für die Medizin. Die Welt, 20.04.2002. Hamburg: Axel Springer Verlag

[6] Schrott E, Ammon HPT. Heilpflanzen der ayurvedischen und westlichen Medizin. Berlin, Heidelberg: Springer; 2012

[7] Köstler U. Pflanzliche Bitterstoffe stärken Lebenskraft und Immunsystem. Akupunktur 2013; 4: 3-5
[8] Benedum J, Loew D, Schilcher H. Arzneipflanzen in der traditionellen Medizin. Bonn: Kooperation Phytopharmaka; 2000

[9] Herrmann K. Über Bitterstoffe in pflanzlichen Lebensmitteln. Dtsch Lebensmittel Rundschau 1972; 68: 105

[10] Saller R, Melzer J, Uehleke B, Rostock M. Phytotherapeutische Bittermittel. Schweiz Z Ganzheitsmed 2009; 21: 200-205. doi: $10.1159 / 000287223$

[11] Meyerhof W, Born S, Brockhoff A, Behrens M. Molecular biology of mammalian bitter taste receptors. A review. Flavour Fragr J 2011; 26: 260-268. doi:10.1002/ff. 2041

[12] Ley JP. Masking bitter taste by molecules. Chemosens Percept 2008; 1: 58-77. doi:10.1007/s12078-008-9008-2

[13] Europäisches Arzneibuch. Stuttgart: Deutscher Apotheker Verlag; 2005

[14] Omer B, Krebs S, Omer H, Noor TO. Steroid-sparing effect of wormwood (Artemisia absinthium) in Crohn's disease: a double-blind placebo-controlled study. Phytomedicine 2007; 14: 87-95. doi:10.1016/j.phymed.2007.01.001

[15] Joy JF, Haber SL. Clinical uses of artichoke leaf extract. Am J Health Syst Pharm 2007; 64: 1904, 1906-1909. doi:10.2146/ ajhp070013

[16] Miccadei S, Di Venere D, Cardinali A et al. Antioxidative and apoptotic properties of polyphenolic extracts from edible part of artichoke (Cynara scolymus L.) on cultured rat hepatocytes and on human hepatoma cells. Nutr Cancer 2008; 60: 276-283. doi:10.1080/01635580801891583

[17] Jachens L. Dermatologie. Berlin: Salumed; 2012

[18] Trivedi BP. Gustatory system: the finer points of taste. Nature 2012; 486: S2-3. doi:10.1038/486S2a

[19] Lindemann B. Receptors and transduction in taste. Nature 2001; 413: 219-225. doi:10.1038/35093032

[20] Chandrashekar J, Mueller KL, Hoon MA et al. T2Rs function as bitter taste receptors. Cell 2000; 100: 703-711

[21] Yarmolinsky DA, Zuker CS, Ryba NJ. Common sense about taste: from mammals to insects. Cell 2009; 139: 234-244. doi:10.1016/j.cell.2009.10.001

[22] Amrein H, Bray S. Bitter-sweet solution in taste transduction. Cell 2003; 112: 283-284

[23] Deshpande DA, Wang WC, Mcllmoyle EL et al. Bitter taste receptors on airway smooth muscle bronchodilate by a localized calcium flux and reverse obstruction. Nat Med 2010; 16: 1299-1304. doi:10.1038/nm.2237

[24] Lee RJ, Xiong G, Kofonow JM et al. T2R38 taste receptor polymorphisms underlie susceptibility to upper respiratory infection. J Clin Invest 2012; 122: 4145-4159. doi:10.1172/JCI64240

[25] Rozengurt E, Sternini C. Taste receptor signaling in the mammalian gut. Curr Opin Pharmacol 2007; 7: 557-562. doi:10.1016/j.coph.2007.10.002

[26] Wölfle U, Elsholz FA, Kersten A et al. Expression and functional activity of the bitter taste receptors TAS2R1 and TAS2R38 in human keratinocytes. Skin Pharmacol Physiol 2015; 28: 137146. doi:10.1159/000367631

[27] Xu J, Cao J, Iguchi $\mathrm{N}$ et al. Functional characterization of bitter-taste receptors expressed in mammalian testis. Mol Hum Reprod 2013; 19: 17-28. doi:10.1093/molehr/gas040

[28] Wölfle U, Haarhaus B, Kersten A et al. Salicin from willow bark can modulate neurite outgrowth in human neuroblastoma SH-SY5Y cells. Phytother Res 2015; 29: 1494-1500. doi:10.1002/ptr.5400

[29] Lu P, Zhang CH, Lifshitz LM, ZhuGe R. Extraoral bitter taste receptors in health and disease. J Gen Physiol 2017; 149: 181-197. doi:10.1085/jgp.201611637 
[30] Wölfle U, Haarhaus B, Seiwerth J et al. The herbal bitter drug Gentiana lutea modulates lipid synthesis in human keratinocytes in vitro and in vivo. Int J Mol Sci 2017; 18 (8): pii: E1814. doi:10.3390/ijms18081814

[31] Lew BL, Cho Y, Kim J et al. Ceramides and cell signaling molecules in psoriatic epidermis: reduced levels of ceramides, PKC-alpha, and JNK. J Korean Med Sci 2006; 21: 95-99. doi:10.3346/jkms.2006.21.1.95

[32] Imokawa G. Lipid abnormalities in atopic dermatitis. J Am Acad Dermatol 2001; 45 (1 Suppl.): S29-S32. doi:10.1067| mjd.2001.117020

[33] Yarosh DB, Both D, Brown D. Liposomal ursolic acid (merotaine) increases ceramides and collagen in human skin. Horm Res 2000; 54: 318-321. doi: 10.1159/000053280
[34] Wölfle U, Haarhaus B, Schempp CM. Amarogentin displays immunomodulatory effects in human mast cells and keratinocytes. Mediators Inflamm 2015; 2015: 630128. doi:10.1155/ 2015/630128

[35] Wölfle U, Haarhaus B, Schempp CM. Bitterstoffe als regenerationsfördernde Wirkstoffe für die Haut. HAUT 2017: 146-148

Bibliografie

DOI https://doi.org/10.1055/a-0654-1711

Zeitschrift für Phytotherapie 2018; 39: 210-215 (c) Georg Thieme Verlag KG Stuttgart · New York ISSN 0722-348X 